\title{
Illness severity measured by CRIB score: a product of changes in perinatal care?
}

\author{
J H Baumer, D Wright, T Mill
}

\begin{abstract}
Aim-To determine the perinatal factors associated with initial illness severity (measured by the CRIB (clinical risk index for babies) score) and its relation to survival to discharge.

Methods-A retrospective study was made of intensive care nursing records on 380 inborn babies, of less than 31 weeks gestation or $1501 \mathrm{~g}$ birthweight, admitted to one unit between 1984-6 and 1991-4.

Results-Between the two time periods mean initial illness severity score increased significantly from 2.8 to 3.9 . This was the result of an increase in the maximum appropriate inspired oxygen concentration in the first 12 hours. Risk adjusted survival did not improve over time after accounting for gestation but was significantly greater after accounting for CRIB score. Illness severity score was also significantly inversely associated with gestation and 1 and 5 minute Apgar scores, using multiple regression analysis. Between the two time periods there was also a $92 \%$ increase in the admission rate of babies under 31 weeks gestation, higher median 1 and 5 minute Apgar scores (6 vs 5 and 9 vs 8 , respectively), more multiple births, and more caesarean section deliveries.

Conclusions-The increase in illness severity score and admission rate may reflect changes in obstetric practice. The increase in illness severity score may also reflect changes in early neonatal care. However, after adjusting for CRIB score, risk adjusted mortality fell significantly, suggesting that neonatal care 12 hours from birth onwards had improved with time.

(Arch Dis Child 1997;77:F211-F215)
\end{abstract}

Keywords: CRIB; illness severity score; Apgar scores; admission rates; multiple births; caesarean deliveries

Paediatrics,

Derriford Hospital,

Plymouth PL6 8DH

Email: harry.baumer@

phnt.swest.nhs.uk

J H Baumer

T Mill

Department of

Statistics,

Plymouth University,

Drake Circus,

Plymouth

D Wright

Correspondence to:

Dr H Baumer.

Accepted 1 May 1997 severity. ${ }^{18}$ For example, the administration of antenatal steroids has been associated with initial illness severity score. ${ }^{9}$ Apparent differences in mortality between centres or within a single centre over time, after adjusting for gestation or birthweight, may reflect differences in these factors rather than changes in the effectiveness of subsequent neonatal care. Adjustment for initial illness severity as in the CRIB score should permit more reliable comparison of the effectiveness of neonatal care after 12 hours.

It has been suggested that the illness severity score of the babies in the initial cohort in the first 12 hours, expressed by these postnatal variables, must have been worse, overall, in tertiary units than in district general hospitals. ${ }^{5}$ This paradoxical finding was unlikely to have been due to poorer early neonatal medical care in tertiary units, in view of the availability of trained medical staff. ${ }^{5}$ To gain the confidence of clinicians, factors affecting a scoring system must be easily understood. ${ }^{10}$ To date, there has been no systematic examination of the factors influencing initial illness severity, using the postnatal components of the CRIB score.

This study examines the association between initial illness severity and perinatal factors and changes in perinatal practice in one district general hospital with a stable population over time. Almost all the neonatal unit's activity is devoted to inborn babies, and very few local preterm infants receive their neonatal intensive care elsewhere. The three postnatal components of the CRIB score obtained in the first 12 hours were examined in two groups of inborn premature infants. Two groups were chosen with a long enough period separating them, in order to identify any association of initial illness severity score with perinatal factors and changes in neonatal care.

\section{Methods}

All inborn infants of less than 31 weeks or less than $1501 \mathrm{~g}$ birthweight were identified from the existing database of premature infants. The first group of 112 babies was born between 1984 and 1986, the second group of 246 between 1991 and 1994. The maximum and minimum appropriate oxygen concentrations and maximum (worst) base deficit in the first 12 hours were abstracted from the nursing intensive care charts. The following information from the database was also checked against the babies' case notes: maternal age; parity and gravidity; whether a singleton pregnancy; whether the baby was malformed; the baby's gestation; birthweight; 1 and 5 minute Apgar scores; type of delivery; sex; first 
temperature after admission to the neonatal unit; and year of birth. The Apgar scores were those assigned at the time, and it was therefore not possible to assess the degree of interobserver variation retrospectively. There was no change in the training of medical or midwifery personnel in the estimation of the Apgar score over the periods of the study. Information was available for $112(95 \%)$ of the 118 infants born between 1984 and 1986, and for 246 (94\%) of 262 of babies born between 1991 and 1994 . These formed the two study groups.

For each baby, the postnatal CRIB score was calculated from the maximum and minimum appropriate inspired oxygen concentration and the maximum base deficit. ${ }^{1}$ Using this as the dependent variable, its association with the other pre- and postnatal factors was analysed using multiple linear regression analysis. Terms not significant at the $5 \%$ level were successively removed using backwards stepwise regression. The association of each component of the postnatal CRIB score with the same terms was also analysed in turn, using the same method. Diagnostic checks were carried out on the residuals obtained from the fitted models. ${ }^{11}$ There was no evidence of any systematic departure from the fitted models nor of any heterogeneity in the variance. However, some of the distributions showed a substantial level of skewness. Changes between the two time periods were assessed, using Student's $t$ test, Mann-Whitney U test or the $\chi^{2}$ test, as appropriate. Logistic regression models were used to predict hospital mortality and were expressed as an area under the receiver operating characteristic (ROC) curve. ${ }^{12}$ The predictive accuracy of CRIB score, birthweight, gestation and the combination of birthweight and gestation were compared. The fit of different logistic regression models was assessed using the deviance statistic. $^{13}$

\section{Results}

One hundred and eighteen inborn babies below 31 weeks gestation or $1501 \mathrm{~g}$ birthweight were admitted to the neonatal unit between 1984 and 1986, compared with 262 babies between 1991 and 1994, despite an overall $6.4 \%$ reduction in birth rate between the two time periods. The admission rate for babies less than 31 weeks gestation increased by $92 \%$ from 5.5 to 10.7 per 1000 births; this increase was seen at all gestations (fig 1 ). There was no significant change in mean birthweight or gestation between the two time periods. There were only two infants with significant congenital malformations in the study.

Survival rates by gestation for the two groups are shown in fig 2 . Increased survival occurred at each gestation up to 28 weeks. This was not significant using a statistical model incorporating gestation, but not the CRIB score, to predict death before discharge (odds ratio $0.79,95 \%$ confidence intervals 0.37 to 1.71 ). When change in survival was tested in a model incorporating the CRIB score, there was evidence of a significant reduction in death before discharge in the most recent group (fig 3); odds

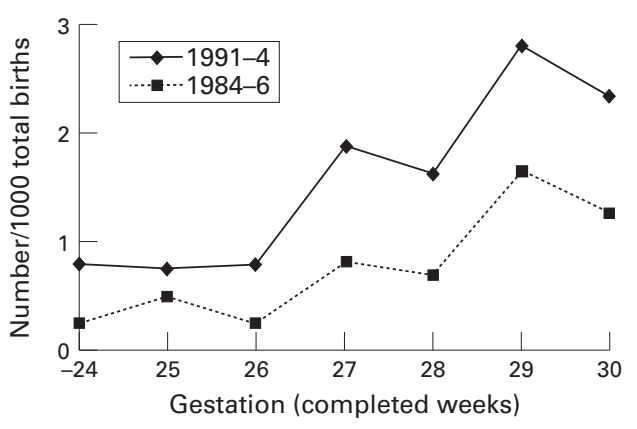

Figure 1 Rate of neonatal admissions by gestation 1984-6 compared with 1991-4.

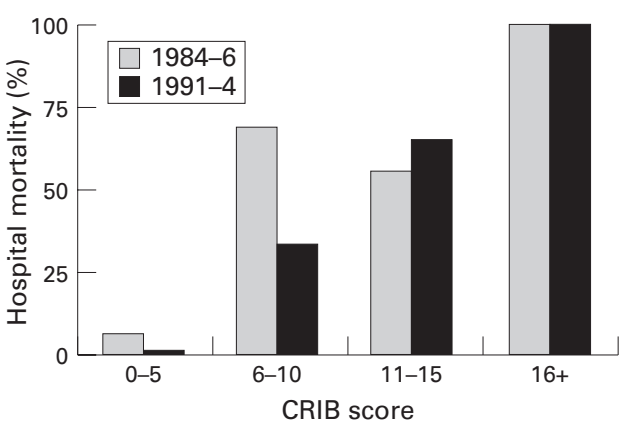

Figure 2 Hospital mortality rates using CRIB score, 1984-6 and 1991-4.

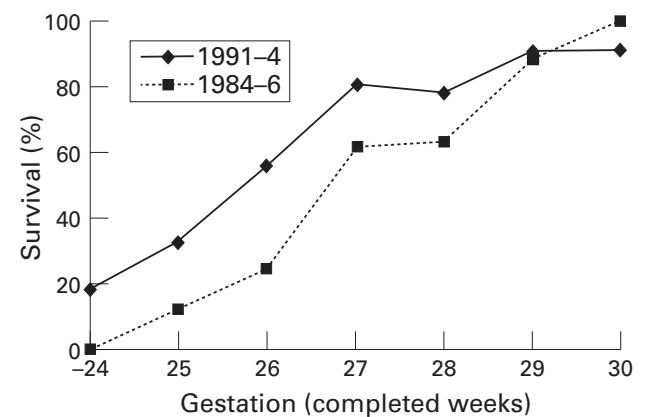

Figure 3 Survival to discharge of babies admitted to neonatal intensive care, 1984-6, compared with those admitted 1991-4.

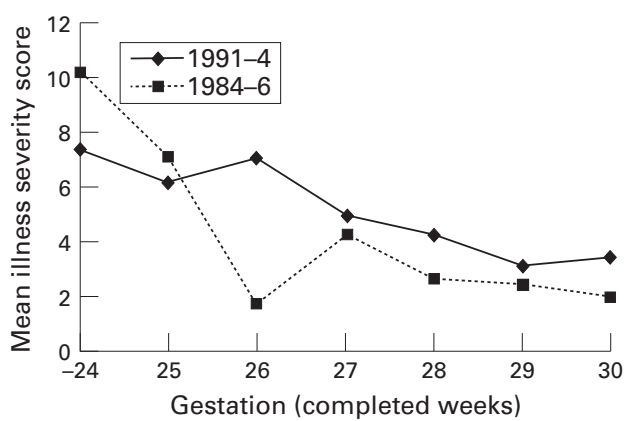

Figure 4 Illness severity scores (measured by CRIB), by gestation, 1984-6 and 1991-4.

ratio for death before discharge for the recent group $0.27,95 \%$, confidence intervals 0.11 to $0.68, \mathrm{p}<0.005)$.

Mean illness severity scores by gestation for each of the two groups are shown in fig 4 . Mean illness severity scores were higher in the 1991-4 group at all gestations above 25 weeks, but lower at 25 weeks and below. Table 1 shows the result of using illness severity score as the 
Table 1 Multiple linear regression model with illness severity as measured by CRIB score as dependent variable

\begin{tabular}{lllcc}
\hline & Coefficient & $S E$ & t test & p Value \\
\hline Gestation (weeks) & -0.45 & 0.066 & -6.9 & $<0.00001$ \\
Time period & 1.36 & 0.36 & 3.8 & 0.0002 \\
Apgar score (5 mins) & -0.35 & 0.11 & -3.3 & 0.001 \\
Apgar score (1 min) & -0.22 & 0.083 & -2.6 & 0.009 \\
Constant & 19.7 & 1.9 & 10.6 & $<0.00001$ \\
\hline
\end{tabular}

dependent variable in a multiple linear regression model. The illness severity score was significantly higher in babies born between 1991 and 1994 than in the earlier group, with a mean score that was 1.36 points higher after the other factors had been taken into account. Gestation and both 1 and 5 minute Apgar scores were the only other factors studied that were significantly associated with illness severity score. Together with year of birth, this accounted for $30 \%$ of the variance in illness severity score. Maternal age, parity and gravidity, mode of delivery, whether a multiple pregnancy, birthweight, sex and admission temperature to the neonatal unit were not significantly associated with illness severity score, when adjusted for the four significant factors.

Median 1 and 5 minute Apgar scores were significantly higher between 1991 and 1994 (median Apgars 5 and 8 in 1984-6 vs 6 and 9 in 1991-4, p<0.001, Mann-Whitney U test). The maximum appropriate inspired oxygen concentration in the first 12 hours after birth was significantly higher between 1991 and 1994 (median $45 \%$ in $1984-6$ vs $64 \%$ in 1991-4, p=0.0004, Mann-Whitney U test). The caesarean section rate increased significantly from $16 \%(18 / 114)$ in $1984-6$ to $58 \%$ $(152 / 261)$ in $\left.1991-4 \chi^{2} 56.0, \mathrm{p}<0.00001\right)$. There was no significant difference between the two time periods in maternal age, parity and gravidity, nor in the baby's admission temperature to the neonatal unit, minimum appropriate inspired oxygen concentration, or maximum base deficit in the first 12 hours.

There was a significantly greater proportion of multiple births during 1991-4 (78 of 261 vs 17 of 118 during 1984-6, $\chi^{2} 9.6, \mathrm{p}<0.005$ ). Although multiple pregnancy was not significantly associated with illness severity score, in multiple pregnancies, allowing for other factors, the firstborns had a significantly lower illness severity score using multiple linear regression $(\mathrm{t}=2.8, \mathrm{p}=0.006)$.

Figure 5 shows receiver operator characteristic (ROC) curves for CRIB score and birth-

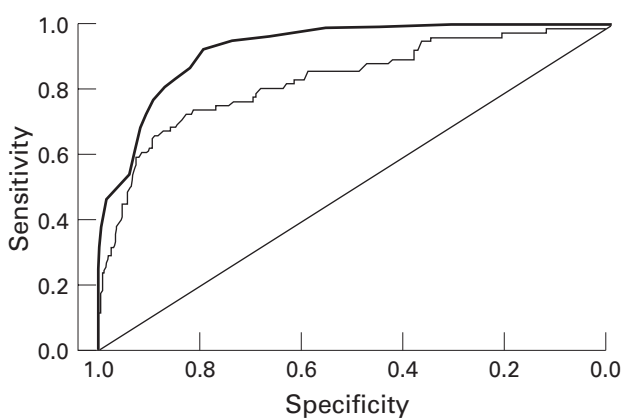

Figure 5 ROC curves for CRIB score (-) and birthweight for prediction of hospital mortality. weight in predicting death before discharge. The best prediction of hospital mortality was obtained using the CRIB score (area under the ROC curve $\mathrm{Az}$ 0.93). Birthweight, gestation, and the combination of the two predicted hospital mortality to a lesser extent (areas under the ROC curve $\mathrm{Az}$ 0.83, 0.83, and 0.85, respectively). A logistic regression model using backward elimination and incorporating all the variables except illness severity was used to optimise the prediction of hospital mortality. Birthweight, gestation, and the 5 minute Apgar score were the only significant factors predicting mortality (area under the ROC curve $\mathrm{Az}$ 0.87).

\section{Discussion}

This study shows a significant increase over time in average illness severity score in the 12 hours after birth. This was associated with improved survival in inborn preterm infants in one maternity hospital. This resulted from higher maximum appropriate inspired oxygen concentrations. There was no evidence of change in the other two components of the score between the two time periods.

Gestation was the best predictor of illness severity score; for every week that gestation decreased, the score increased by 0.45 , after adjustment for other factors. All three individual components of the illness severity score were strongly associated with gestation. There was no difference in the gestation of babies born during the two time periods, and this does not explain the increase in illness severity score.

One minute Apgar scores, reflecting the condition of the baby at birth, were inversely associated with illness severity, higher maximum appropriate inspired oxygen concentrations, and maximum base deficit in the first 12 hours. The increase in the caesarean section rate suggests a more aggressive obstetric policy in managing problems below 31 weeks of gestation. The higher 1 minute Apgar scores in the recent group may reflect improved pulmonary maturity, but these are also consistent with a reduction in intrapartum asphyxia associated with improved obstetric care. However, the possibility of significant bias in the estimation of Apgar scores between the two time periods cannot be excluded.

Five minute Apgar scores were inversely associated with illness severity, minimum appropriate oxygen concentrations, and with the maximum base deficit in the first 12 hours. In the absence of information about illness severity, the 5 minute Apgar score also contributed to the prediction of hospital mortality together with birthweight and gestation. If the increase in 5 minute Apgar scores between the two time periods is real, it may reflect improved obstetric care. Equally, however, it may indicate an improvement in one important aspect of neonatal care contributing to illness severity-the initial resuscitation of the baby at birth.

Despite advances in antenatal and neonatal care between the two time periods, mortality did not improve significantly after adjusting for gestation, in contrast to the significant 
improvement after adjusting for CRIB score. The improvement is consistent with the introduction of proved advances in neonatal care beyond 12 hours from birth. For example, the introduction of surfactant replacement (the synthetic surfactants Exosurf then ALEC were introduced during the second period) would be expected to result in improved survival, after initial illness severity is captured by CRIB. The failure to show a significant improvement in gestation specific mortality over time may simply reflect inadequate sample size, as evidenced by the wide confidence intervals. Routine ultrasound assessment of gestation before 20 weeks was available throughout both time periods. Differences in the accuracy of measurement of gestation between the two time periods could have occurred as a result of improvements in real time ultrasound scanners after 1986. Although possible, it seems unlikely that this would have led to bias resulting from systematic overestimation of gestation in the earlier period.

When the illness severity score is used together with birthweight and gestation in the full CRIB score, there is a much stronger weighting for birthweight than gestation. This seems to contradict the findings of VerlooveVanhorick et al, ${ }^{14}$ who found that gestation was more strongly associated with birthweight than survival. The present study confirms the superior ability of CRIB score to predict hospital mortality over birthweight alone. Gestation predicted hospital mortality to a similar extent to birthweight, but the CRIB score was still better than the combination of birthweight and gestation for predicting hospital mortality. We suggest that the reason why gestation has such a small weighting in the CRIB score is that it exerts its influence on survival to discharge mainly through the illness severity component of the CRIB score. The nature of the postnatal components of the CRIB score, combining oxygen requirements and degree of metabolic acidosis, suggests that gestation affects survival by its association with pulmonary immaturity and the severity of perinatal asphyxia. Birthweight, by contrast, may affect survival to discharge by other mechanisms.

While neither the perinatal variables associated with illness severity, nor minimum appropriate oxygen concentration, nor worst base deficit were significantly worse in the second time period, this may simply reflect a type II error owing to inadequate numbers. Throughout the two time periods, the policy was to transfer all liveborn very low birthweight infants above 23 weeks gestation to the neonatal unit. The maternity unit continued to provide a service for the local population with only a very small number of in utero referrals into or out of the hospital. One possible explanation for the increased rate of admissions to the neonatal unit between the two time periods is that some of the babies admitted to the neonatal unit in 1991-4 would have died in utero between 1984 and 1986. More functional immaturity among these survivors might account for the higher postnatal CRIB scores in the more recent group of babies, despite their better 1 minute Apgar scores. If this is the explanation, this study confirms the importance of using the postnatal CRIB score to adjust for differences in clinical severity when comparing the effectiveness of different neonatal units. It is, however, quite possible that the higher maximum appropriate inspired oxygen concentrations, unaccompanied by any change in the minimum appropriate inspired oxygen concentration, may reflect aspects of early postnatal care, such as suboptimal fluid or ventilatory management. Even if this were the case, the improvement in mortality over time after adjusting for CRIB suggests that neonatal care beyond 12 hours after birth was more effective in the second time period. This illustrates the value of using illness severity measured shortly after birth to assess risk adjusted outcome.

Alternatively, changes in oxygen monitoring or administration could affect the observed maximum appropriate inspired oxygen concentration between the two time periods. If so, the apparent increase in illness severity may reflect a measurement artefact. This emphasises the need for accurate standardisation of respiratory variables and all measures of risk, such as gestation and birthweight, when comparing risk adjusted outcomes. Measurement artefact is less likely if neonatal units have instruments which measure arterial and administered oxygen calibrated and maintained by a medical physics department, as was the case in this study. If either suboptimal initial management of fluids or ventilation, or changes in the recording of oxygen administration is the true explanation for the higher illness severity scores over time, then the postnatal CRIB score may be concealing important avoidable factors affecting the condition of babies, or giving an inappropriate indication of improved neonatal management.

In babies below 26 weeks gestation there was a trend towards lower mean postnatal CRIB scores in the more recent babies (fig 4). Improvements in initial care for the most immature babies may have improved their initial clinical state in contrast to the larger numbers of more mature babies in whom improved survival over time was associated with higher postnatal CRIB scores.

The nearly fourfold increase in the rate of multiple pregnancies is unlikely to be explained wholly by an increased readiness to deliver babies before 31 weeks of gestation; the finding therefore confirms the impact of assisted fertility treatments on the population of very low birthweight babies and on the neonatal service. ${ }^{15}{ }^{16}$ Although multiple birth had no significant effect on postnatal CRIB score or survival, firstborn babies had significantly lower postnatal CRIB scores, perhaps reflecting greater pulmonary maturity and reduced intrapartum asphyxia. There have been conflicting reports of the effects of birth order on survival to discharge in multiple births. ${ }^{17}{ }^{18}$ In this study there was no effect of birth order on survival to discharge after adjustment for CRIB score.

In conclusion, this study shows that illness severity, as measured by the CRIB score, is 
strongly associated with gestation and both 1 and 5 minute Apgar scores. The increase in illness severity between the two time periods was the result of higher maximum appropriate inspired oxygen concentrations without changes in the other two components of the score. This increase could reflect changes in obstetric practice and in early neonatal care. Despite the increase in illness severity, after adjustment for CRIB score, survival significantly increased, suggesting that neonatal care 12 hours from birth onwards became significantly more effective between the two time periods, in keeping with the introduction of advances in neonatal care. CRIB may be a valuable tool for interpreting patterns of hospital mortality within a single hospital.

1 International Neonatal Network. The CRIB (clinical risk index for babies) score: a tool for assessing initial neonatal risk and comparing the performance of neonatal intensive care units. Lancet 1993;342:193-8.

2 de Courcy-Wheeler RHB, Wolfe CDA, Fitzgerald A, Spencer $M$, Goodman JDS, Gamsu HR. Use of the CRIB (clinical risk index for babies) score in prediction of neonatal mortality and morbidity. Arch Dis Child 1995;73:F32-F6.

3 Scottish Neonatal Consultants' Collaborative Study Group and the International Neonatal Network. CRIB (clinical risk index for babies), mortality, and impairment after neonatal intensive care. Lancet 1995;345:1020-2.
4 Grant JM. The CRIB score. Lancet 1993;342:612.

5 Hope P. CRIB, son of Apgar, brother to APACHE. Arch Dis Child 1995;72:F81-F3.

6 Hughes-Davies TH. The CRIB score. Lancet 1993;342:938.

7 Tarnow-Mordi W, Ogston S, Wilkinson AR, et al. Predicting death from initial disease severity in very low birthweight infants: a method for comparing the performance of neonatal units. BMF 1990;300:1611-14.

8 Richardson DK, Tarnow-Mordi WO. Measuring illness severity in newborn intensive care. $\mathcal{F}$ Intensive Care Med 1994;9:20-33.

9 Tarnow-Mordi W, Cooke R, Parry G, Ogston S. Authors' reply. Lancet 1993;342:613.

10 Wyatt JC, Altman DG. Commentary: Prognostic models: clinically useful or quickly forgotten? $B M \mathcal{F}$ 1995;311:1539-41

11 Draper NR, Smith H. Applied regression analysis. New York: John Wiley and Sons, 1981 .

12 Hanley JA, McNeill BJ. A method of comparing the areas under the receiver operating characteristic curves derived from the same cases. Radiology 1983;148:839-43.

13 McCullagh P, Nelder JA. Generalised linear models. 2nd edn. London: Chapman and Hall, 1989.

14 Verloove-Vanhorick SP, Verwey RA, Brand R, Bennebroek Gravenhorst J, Keirse MJNC, Ruys JH. Neonatal mortality risk in relation to gestational age and birthweight. Lancet 1986 ; $\mathbf{i}: 55-7$.

15 Kiely JL, Kleinman JC, Kiely M. Triplets and higher-order multiple births. Time trends and infant mortality. $A m \mathcal{F} D i$ s Child 1992;146:862-8

16 Seoud MA, Toner JP, Kruithoff C, Muasher SJ. Outcome of twin, triplet, and quadruplet in vitro fertilization pregnancies: the Norfolk experience. Fertil Steril 1992;57:825-34.

17 Chen SJ, Vohr BR, Oh W. Effects of birth order, gender, and intrauterine growth retardation on the outcome of very intrauterine growth retardation on the outcome of
low birth weight in twins. $\mathcal{F}$ Pediatr 1993;123:132-6.

18 Imaizumi Y. Perinatal mortality in single and multiple births in Japan, 1990-1991. Paediatric Perinatal Epidemiol 1994;8:205-15 